\title{
Defects in DNA replication hit NK cells and neutrophils
}

\author{
Klaus Ley
}

Division of Inflammation Biology, La Jolla Institute for Allergy and Immunology, La jolla, California, USA.

\begin{abstract}
Patients who present with unique immunological phenotypes provide an opportunity to better understand defect-driving mutations. In this issue of the $J C l$, Cottineau and colleagues characterize 5 individuals who exhibited growth restriction, facial deformities, and a history of bacterial and viral infection. Further characterization revealed that these patients were neutropenic and NK cell deficient. These phenotypes were unexpectedly linked to mutations in the gene encoding a subunit of the Go-Ichi-Ni-San (CINS) complex, which is essential for DNA replication prior to cell division. Together, the results of this study lay the groundwork for future studies to explore the role of DNA replication in immune cell generation and function.
\end{abstract}

\section{Unexpected cause of NK cell deficiency and neutropenia}

In this issue, Cottineau and colleagues (1) report the characterization of 5 patients with previously unidentified mutations in the 5' UTR of the gene encoding Go-IchiNi-San (GINS) complex subunit 1 (GINS1). GINS1 is a required component of the GINS complex, which plays an essential role in DNA replication preceding cell division $(2,3)$. As expected for such a fundamental defect, the patients experienced a wide range of conditions, including intrauterine and postnatal growth retardation, facial deformities, and immunological defects. Unexpectedly, these patients also completely lacked natural killer (NK) cells and suffered from neutropenia. These immune defects resulted in viral and bacterial infections, osteosarcoma, protein-losing enteropathy, hypothyroidism, premature aging, and autoimmune hemolytic anemia.

The GINS complex is a heterodimer composed of GINS1, GINS2, GINS3, and GINS4. At the $\mathrm{G}_{1}$-to-S phase transition, the GINS complex is recruited to the origins of replication. The mutations identified by Cottineau and colleagues in the 5' UTR mutant of GINS1 resulted in reduced expression and deletions in exon 2. However, in these patients, about $5 \%$ to $10 \%$ of WT GINS1 was still transcribed. This residual WT GINS expression may explain the phenotype of these patients, as global Gins1 deletion in mice results in embryonic lethality (4).

Cottineau et al. determined that the amount of GINS1 expressed in patientderived cells ranges from $29 \%$ to $53 \%$ of the level of GINS1 generated by cells from healthy controls (1). This reduced expression apparently leads to decreased expression of GINS3 and GINS4, resulting in defective GINS complex assembly. Consistent with these findings, fewer patientderived fibroblasts were found to be in $G_{1}$, and they were more likely to be in $G_{2} / M$, resulting in slower proliferation and signs of senescence. The slow replication was caused by fewer replication clusters and fewer bidirectional replication forks per cell, defects that were partially compensated by an increase in replication speed compared with control-derived fibroblasts.

Why are neutrophils and NK cells so severely affected by the GINS1 mutations? It could be argued that neutrophil precursors must divide a lot to supply the billions of neutrophils that are needed each day. Thus, neutrophil precursors might be severely affected by a DNA replication defect. NK cell precursors on the other hand would not be expected to divide as much, because NK cells are a

Related Article: p. 1991

Conflict of interest: The author has declared that no conflict of interest exists.

Reference information: / Clin Invest. 2017;127(5):1616-1617. https://doi.org/10.1172/JCI93835.

longer-lived population, and, therefore, fewer precursors are needed each day to replenish the pool. Unfortunately, at this time there is not really a unifying hypothesis explaining the phenotypes of the patients.

Treatment efforts in these patients may provide a clue as to how GINS1 deficiency affects NK cell and neutrophil populations. In one of these patients, the neutropenia was rescued by granulocyte colony-stimulating factor (G-CSF), a cytokine that speeds up neutrophil maturation. However, the addition of IL-2 or IL-15 to patient PBMCs did not rescue the NK cell defect. It seems reasonable that NK cells may be particularly susceptible to elimination if replication is defective or imprecise. Such a notion would make biological sense, because aberrant NK cells could be very dangerous to the host. However, it is unclear what the elimination mechanism might be, and how replication defects would be detected.

\section{Concluding remarks}

Overall, this is an exciting and provocative article from Cottineau and colleagues. Like any new discovery, it raises more questions than it answers. More work will be required to determine the mechanism by which GINS1 defects affect neutrophils and NK cells and why these mutations do not seem to affect $\mathrm{T}$ or B lymphocytes or monocytes. It may be useful to generate a hypomorphic Gins1 mutant mouse by gene targeting and homologous recombination or by CRISPR to better address how GINS1 deficiency influences different cell populations. Such a mouse line might produce live litters in which expected neutrophil and NK cell defects could be studied.

\section{Acknowledgments}

The author was supported for neutrophil work by NIH grant P01 HL078784.

Address correspondence to: Klaus Ley, La Jolla Institute for Allergy and Immunology, 9420 Athena Circle Drive, La Jolla, California 92037, USA. Phone: 858.752.6661; E-mail:klaus@lji.org. 
1. Cottineau J, et al. Inherited GINS1 deficiency underlies growth retardation along with neutropenia and NK cell deficiency. J Clin Invest. 2017; 127(5):1991-2006.

2. Gambus A, et al. GINS maintains association of
Cdc45 with MCM in replisome progression complexes at eukaryotic DNA replication forks. Nat Cell Biol. 2006;8(4):358-366.

3. Ilves I, Petojevic T, Pesavento JJ, Botchan MR. Activation of the MCM2-7 helicase by associ- ation with Cdc 45 and GINS proteins. Mol Cell. 2010;37(2):247-258.

4. Ueno M, Itoh M, Kong L, Sugihara K, Asano M, Takakura N. PSF1 is essential for early embryogenesis in mice. Mol Cell Biol. 2005;25(23):10528-10532. 